\title{
Mobile Agent based System for Listing Fundamental Wi-Fi Peer-to-Peer Network Details
}

\author{
Kevin Otieno Gogo \\ Senior lecturer, Kenya School of Government, \\ and County ICT Manager -Tharaka Nithi County \\ Government \\ P.O Box 17421 - 00100 Nairobi
}

\author{
Elisha Toyne Opiyo \\ Senior lecturer, School of Computing and \\ Informatics \\ University of Nairobi \\ Box 30197 - 00100 GPO Nairobi
}

\begin{abstract}
On our everyday operations there is need to engage agents to perform some duties on our behalf, hence they are gaining acceptance as a technology and are being used. Most of the networked offices, networked homes, cyber cafes, learning institutions and other arenas where computers are interconnected on a Wi-Fi network, have peer-to-peer networks. In Wi-Fi peer-to-peer networks, it is difficult to identify the network details of all the network devices connected such as the IP addresses, Mac addresses and computer names of all computers connected on the Wi-Fi peer to peer network at one go; which we hereby refer to as fundamental network details. This is mainly possible in a client-server based architecture where the server monitors all the computers on the network. From the above gap, we developed a mobile agent that could be run in any computer on the Wi-Fi peer-to-peer network and it lists these fundamental details of all the computers connected to the Wi-Fi peer-to-peer network. In developing this mobile agent, we used the MaSE agent methodology. The mobile agent was coded, implemented and tested. The agent was then subjected to various controls which it overcame and managed to return the desired fundamental details with over $80 \%$ accuracy. They had the capacity to classify every computer on the network as either intruder or non intruder based on the list of authorized computers supplied by the user. The agent suffered major limitation which included: - the agent took longer time to learn and return the results, as well as it could not communicate to the intruders or shut them down. In future, the agent could therefore be improved to reduce its processing time, communicate with the intruding computers, and shut down the intruding computers or deny them network access.
\end{abstract}

\section{General Terms}

Multiagent systems, computer networking

\section{Keywords:}

Mobile agents, agents programming, Wi-Fi networks, peer networks.

\section{INTRODUCTION}

On our everyday operations there is need to engage agents to perform some duties on our behalf, hence they are gaining acceptance as a technology and are being used. There is a growing need for practical methods for developing agent applications to ease some of our duties. [16] suggests that agents in multi-agents systems setup are usually concurrent autonomous entities that coordinate and cooperate so as to perform their various tasks, these coordination and cooperation tasks might be achieved through communication, interaction etc.

Mobile agent based system for listing fundamental Wi-Fi peer-to-peer network details is an agent that is responsible for listing details of the computers found on the Wi-Fi P2P network or workgroup. It lists IP addresses, Mac addresses and computer names of the computers. There being various categories of computer networks, this agent is best used in a small Wi-Fi WLAN environments such as in the small cybercafés, schools, networked offices, wireless home networks and other small Wi-Fi P2P computer networks. This agent will be useful to the computer users and the network administrators as it will be able to monitor who are the Wi-Fi P2P network users, hence discouraging the illegal network users who do piggybacking on unauthorized Wi-Fi networks. This could be further attributed to the fact that most home computer networks today are Wi-Fi P2P networks.

\subsection{Problem Statement}

The primary goal of this study is to design and build a fundamental network details listing agent using jade platform, then implement, test and evaluate the agent, with a view to return the fundamental Wi-Fi peer-to-peer network details of the devices connected to that network. The agent also classifies the devices connected on the $\mathrm{Wi}$ Fi P2P network as either intruders or non-intruders to the LAN, based on their IP addresses and could be run on any computer on the Wi-Fi P2P network.

\subsection{Research Objectives}

The research objectives of this project are as follows:-

- To design and build a fundamental network details listing agent for Wi-Fi P2P network using the java platform

- To implement, test, and evaluate the fundamental network details listing agent on an existing Wi-Fi P2P network configuration

\section{RELATED TECHNOLOGIES AND LITERATURE REVIEW}

\subsection{Related Technologies}

There are quite a number of related technologies in line with our system in the field of computing. Some of the related technologies and solutions include: -

\subsubsection{Network Management Protocols}

Arguments by [17] and [18] illustrates that a set of automated network management tools usually deals with the multi-vendor environment of the typical installation; a 
network-management system is based on standardized network management protocols and applications which they are designed to achieve. Due to advanced technology, a large number of network management protocols exist to support network and network device management, although majority of them are not readily available to be used as a package on Wi-Fi peer to peer networks for network details listings. Some of these network management protocols which are highly in line with this project include and not limited to:
a. Simple Network Management Protocol (SNMP),
b. Common Management Information Protocol (CMIP),
c. XML-based network management.

2.1.3 Microsoft Windows Management Instrumentation (WMI)

The WMI technology is the Microsoft implementation of the Distributed Management Task Force (DMTF) WebBased Enterprise Management (WBEM) initiative that extends the Common Information Model (CIM) to represent management objects in Windows-based management environments. The Common Information Model, also a DMTF standard, is an extensible data model for logically organizing management objects in a consistent, unified manner in a managed environment [8]. Based on the Common Information Model, WBEM is a DMTF initiative and technology that establishes management infrastructure standards and provides a standardized way to access information from various hardware and software management systems in an enterprise environment. WBEM provides a point of integration through which data from management sources can be accessed.

\subsubsection{Peer-to-peer File sharing software system}

Some of these file sharing software like Kazaa and Napster rank amongst the most popular software applications enable the exchange of some network information across the network, as well as the music and video files across the networks. Although majority of these file sharing software do not disclose the fundamental network details of the all the devices connected on a Wi-Fi peer to peer networks [15].

\subsection{Literature review}

\subsubsection{Computer networks and the IP addresses}

Computer network speeds have also increased, which has made it possible to exchange information around the world in a much more feasible manner; in view of this we now hear and read about globalization where the world is tending to become one global village [11]. For the computers on the network to communicate to each other there has to be some set of rules which have to be followed to effect communication, these rules are referred to as the Internet Protocol (IP). There are various computer networks namely: - Local area networks (LAN), Metropolitan area networks (MAN), wide area networks (WAN), virtual area networks (VAN), and personal area network (PAN).

IP address is an exclusive number that all information technology devices (printers, routers, modems, etc) use which identifies and allows them the ability to communicate with each other on a computer network. The IP address could be either Static IP or a dynamic IP. The IP addresses are moving from IP version 4 (IPv4) currently used by most network devices to IP version 6 (IPv6), the move is due to more and more computers accessing the internet [2]

\subsubsection{Peer-to-peer / Ad-hoc Networks}

Peer-to-peer connection is a connection where any computer on the network can be a client or a server or both at the same time, i.e. there is no dedicated server to control the clients. P2P networking type is most commonly used computer networks [10]. This network type is very cost effective but supports lesser number of computers in network, about ten to fifteen computers can be connected to each other using $\mathrm{P} 2 \mathrm{P}$ networking model without problem, more number of computers often create problems.

\subsubsection{Wi-Fi Networks}

$\mathrm{Wi}-\mathrm{Fi}$ is a communication technology that uses the 'Directsequence spread spectrum radio technology' and the 'Orthogonal Frequency Division Multiplexing radio technology', Wi-Fi is the trademark used by a trade group known as Wi-Fi Alliance. Wi-Fi networks Operating in peer-to-peer mode allows all wireless devices within range of each other to discover and communicate in ad-hoc fashion without involving central access points including those built in to broadband wireless routers [7]. Below is a figure 1 showing Wi-Fi peer-to-peer network.

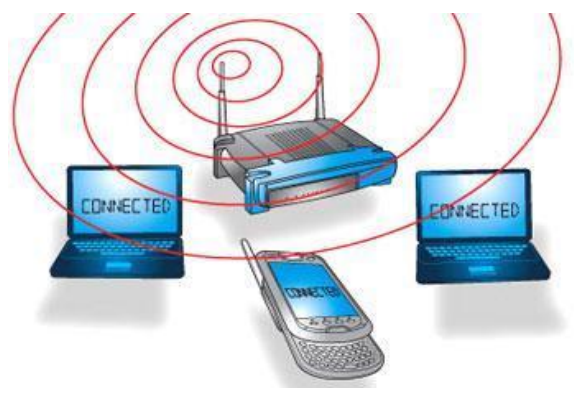

Figure 1: Wi-Fi peer-to-peer network

Source: [3]

\subsubsection{Agents}

Agents are autonomous programs that can perform services on behalf of the user. Agent designing is taskoriented. Instead of looking at what actors are involved in an operation, you look at what tasks and subtasks that the operation is consisting of, agents are then created to solve these tasks. Whereas object orientation does not say anything about the actual tasks but rather expects the objects to solve them implicitly, agent orientation concentrates on the tasks at hand and creates actors that can help in solving these tasks [9] and [16]. There are various categories of agents as illustrated below: -

\subsubsection{Network Agents}

There are a numerous network agents that have been designed and are in existence, but a majority of the agents are biased towards network security, client/server networks among others. Most of the network details listing agents are embedded within the operating systems, but this is merely to list only a few network details such as the IP address of the computer that the operating system is running within [10]. Network features, such as Network 
card speed, Network switch speed, etc., does impact the network throughput of these agents.

\subsubsection{Mobile Agents}

Mobile Agents are programs that can migrate from host to host in a network. They should be able to execute on every machine in a network and the agent code should not have to be installed on every machine the agent could visit. Therefore Mobile Agents use mobile code systems like Java and the Java virtual machine where classes can be loaded at runtime over the network [6] and [12]. Below is figure 2 showing a mobile agent movement.

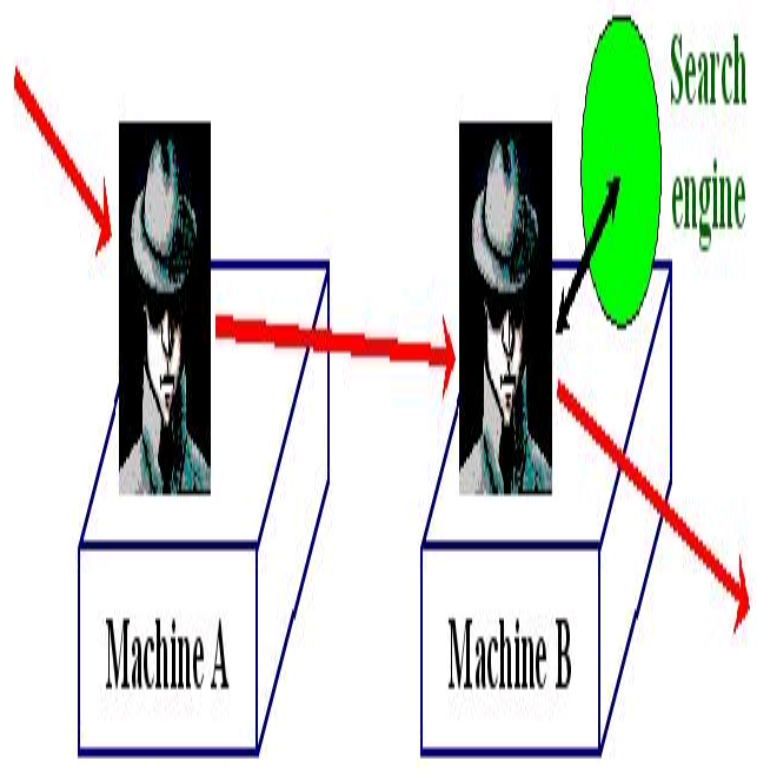

Figure 2: Mobile agent movement illustration

Source: [12]

Mobile agents are seen as a potential threat to systems. If mobile agents were misused they could generate denial of service attacks or even steal data. Most mobile agents are heterogeneous so that they can travel around a heterogeneous network; hence they have all the characteristics of an agent. Mobile agents send not only data, but code, hence more network bandwidth is required. This is often offset or even reduced to lower level than without mobile agents by the fact that data can be processed at various locations in the network [6].

\subsubsection{Mobile Agents Programming}

As suggested by [14] and [5], there are various programming languages that can be used in implementing mobile agents, as long as the language has the following capabilities: - support for agent migration, support for agent-to-agent communication, support for interaction with local resources, security mechanisms, suitable execution efficiency, language implementation across multiple platforms, and Ease of programming of the tasks mobile agents perform. We used jade programming language in coding our agent, though there are other agent programming languages such as aglets, odyssey, Voyager, telescript, oblique and perl5 among others.

\section{METHODOLOGY}

\subsection{Systems Design}

In system designs as advised [1], we used MaSE (multi agent system engineering) methodology. This methodology advocates for two phases namely analysis phase and designing face. In analysis phase we captured the agent's goals, applying use cases and refine agent's roles. While in designing phase we created the agents classes, assemble agent's classes then finally design the agent, as shown in table 1 below.

Table 1: MaSE methodology steps

\begin{tabular}{|c|c|}
\hline$\underline{\text { Models }}$ & Phase \\
\hline \multirow{5}{*}{$\begin{array}{l}\text { Goal Hierarchy } \\
\text { Use Cases, Sequence } \\
\text { Diagrams } \\
\text { Concurrent Tasks, Role } \\
\text { Model }\end{array}$} & 1) Analysis phase \\
\hline & a) Capturing goals \\
\hline & b) Applying use cases \\
\hline & \multirow{2}{*}{ c) Refining roles } \\
\hline & \\
\hline \multirow{6}{*}{$\begin{array}{l}\text { Agent class diagrams } \\
\text { Conversation diagrams } \\
\text { Agent Architecture Diagrams } \\
\text { Deployment Diagrams }\end{array}$} & 2) Design phase \\
\hline & a) Creating agent \\
\hline & Hasses \\
\hline & $\begin{array}{l}\text { b) Constructing } \\
\text { conversations }\end{array}$ \\
\hline & $\begin{array}{l}\text { c) Assembling agent } \\
\text { classes }\end{array}$ \\
\hline & d) System design \\
\hline
\end{tabular}

3.2 Experimental and data sources design

We began by setting up a Wi-Fi P2P network. In setting up this network we send the Wi-Fi signals from a wireless router or joined a P2P WLAN hotspot. We then ran our prototype in any randomly sampled computers forming the Wi-Fi P2P network and documented the results. We also added and removed other computers on the network and ran the prototype, then recorded the results obtained. The recorded results constituted the data which we used for the analysis of the mobile agent.

\subsection{Data collections procedures, methods and tools}

In data collections as argued by [4], in our project we collected the data through experiments and observations methods. The data was recorded at source, immediately they were collected.

\subsection{System implementation and testing}

In the implementation of the fundamental network details listing agent, first we had to setup a Wi-Fi P2P network. In setting a P2P network, we either used Wi-Fi. Then we installed JAVA, this is because we are using java based framework. We then ran the system by invoking the agent.

\subsection{Data analysis and Evaluation}

In analysis we printed the screen shots of the results. We analyzed and evaluated the data with regard to real life experience and some sets of controls. The analysis and evaluations was done in a way such that we compared the data that the agent gave under various tests, and the actual information on the ground. Some of these comparisons were; how many fundamental computer network details does the agent return, while actually how many computers are connected to the Wi-Fi P2P network, classifying the computers as either allowed or intruder etc. 


\section{IMPLEMENTATION \\ 4.1 Conceptual framework}

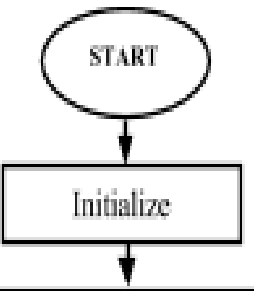

Mobile agent scans and identifies hosts on the Wi-Fi P2P Network

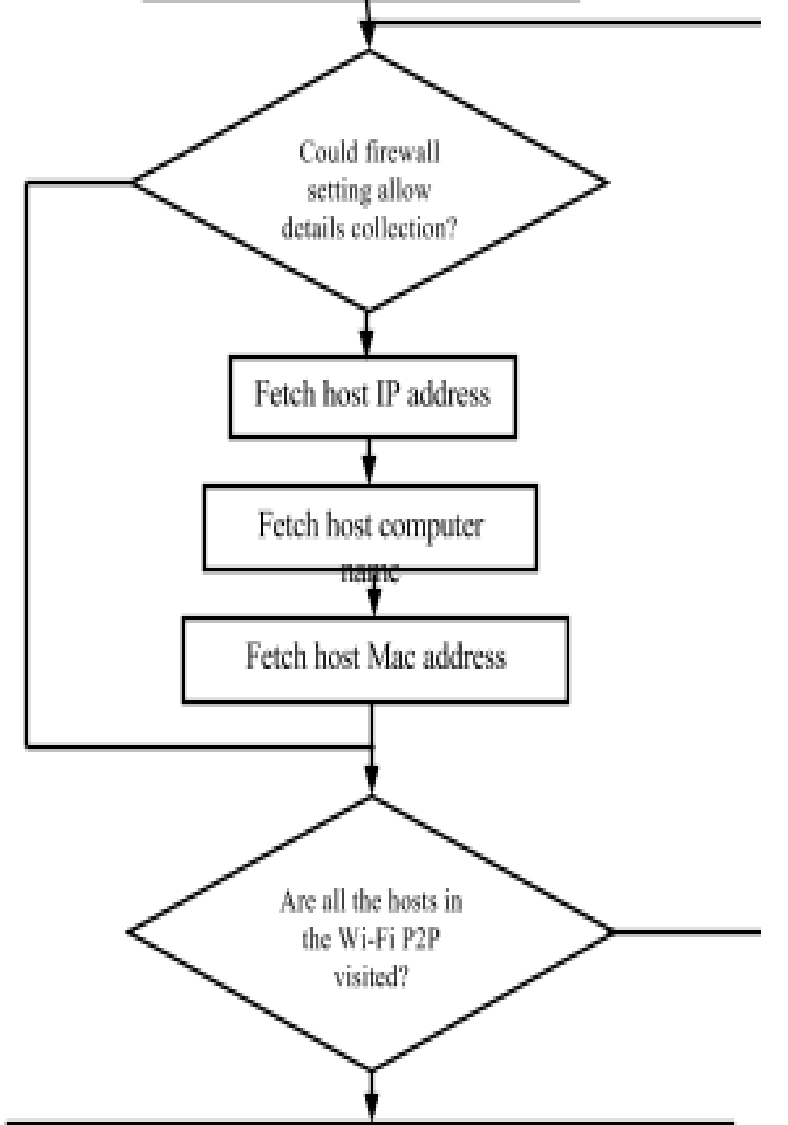

Enter the IP addresses of non-intruding computers on a file

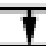

Compares the found IPs and the non-intruding IPs from the file

\section{$\uparrow$}

Display all the fetched details, notifying on non-intruding computer details, and illegally intruding computers.

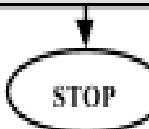

\subsection{Sample Code for Analysis Agent}

This is a sample code for the analysis agent, which analyses the IPs from the network scan agent and the allowed IPs file, and compares them to classify the computers as either intruding or non-intruding.

package agents; import abstracts.AbstractAgent;

import behaviours.ReceiveMessages;

// @author gogo

public class AnalysisAgent extends AbstractAgent'

$$
\text { @ Override }
$$

protected void setup()

l

register("Analysis Agent");

addBehaviour(new ReceiveMessages(this));

\}

\}

\subsection{Sample Code for Network Scanner}

This is a sample code that scans the Wi-Fi P2P network and returns the network details that have been identified and forwards them to the analysis agent. It's in this code that one can change the range of IPs to be scanned.

/*

* To change this template, choose Tools $\mid$ Templates and open the template in the editor.

$* /$

package agents;

import abstracts.AbstractAgent;

import behaviours. GetAllIps;

//@author gogo

public class NetworkScanner extends AbstractAgent \{

\section{@ Override}

protected void setup () (

register("Network Scanner");

int range $=2 ; \quad / / N o$ of machines scanned by one behavior

$$
\begin{gathered}
\text { for }(\text { int } i=1 ; i<20 ;)\{ \\
\text { int pass }=((i+\text { range })-1) ; \\
\text { if }(\text { pass }>254) \\
\text { pass }=254 ;
\end{gathered}
$$

addBehaviour(new GetAllIps(this, i, pass));

//System.out.println( $(i+"$ - "+((i+range $)-1))$;

$i+=$ range;

\}

\}

\}

\section{TESTING AND RESULTS}

\subsection{1 testing the system with no internet and no IP given as allowed}

When the system was tested without internet but with networked computers and no internet, the following result was obtained as shown in figure 3 below: 


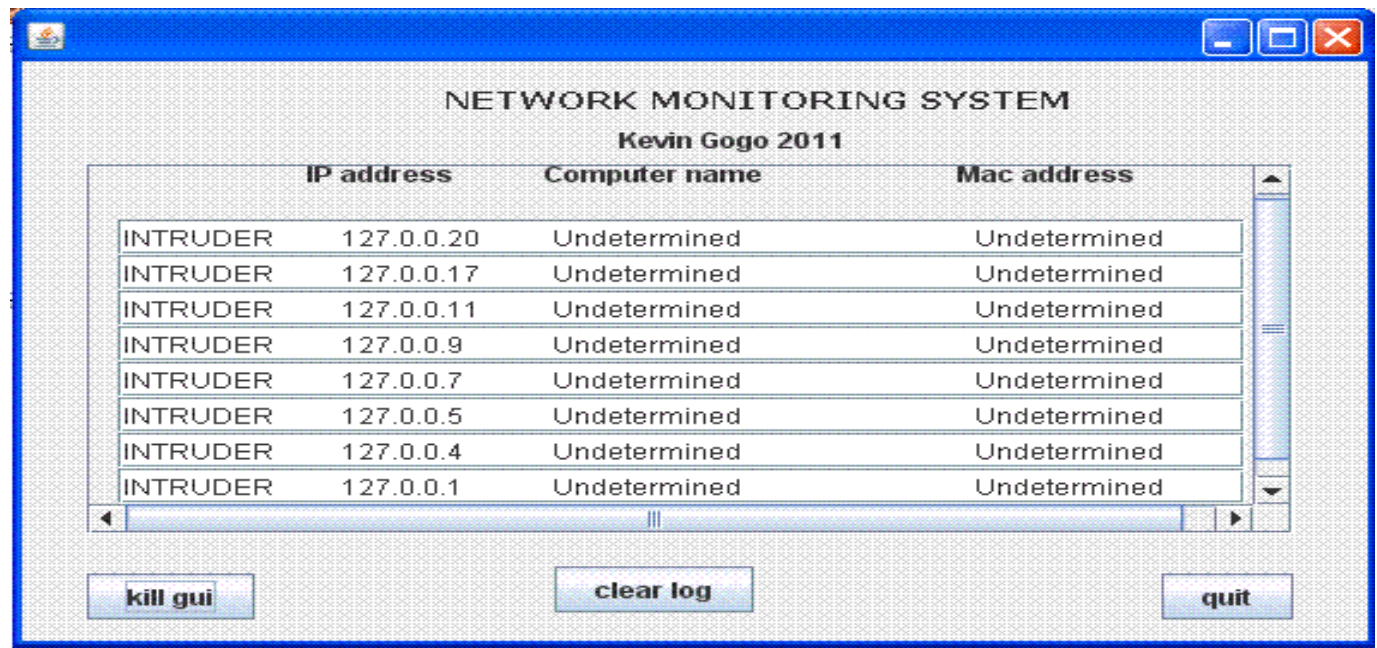

Figure 3: Systems GUI showing results when no internet and no allowed IPs given

\subsection{Testing the System when Connected to a Network and no Allowed IPs Entered}

When the system was connected to a Wi-Fi network connecting 4 computers without computer names, it returned the details of the 3 computers connected to the Wi-Fi network, missing one computer.
It managed to classify all the IPs of the detected computers as INTRUDING, this is because we did not entered any computer IP address on the allowed IP file and so any detected IP will be classified as INTRUDING. Figure 4 below shows the systems interactive GUI with the results.

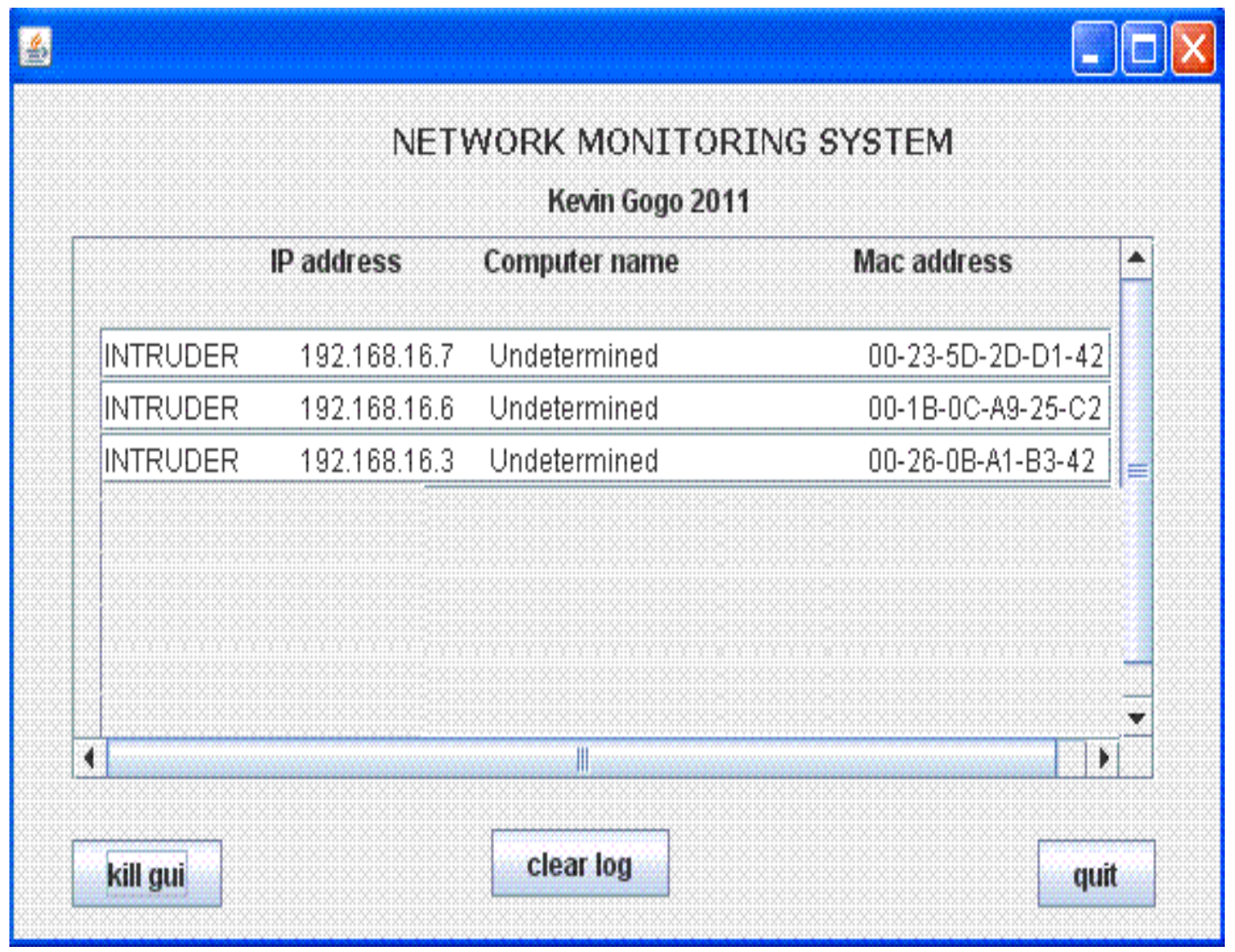

Figure 4: Systems GUI showing results when there is network but with no allowed IPs given

\subsection{Testing the System when Connected to a Network with Allowed IPs Entered}

The system was made to continue running this time with allowed IPs of (41.89.64.77) and (41.89.64.74)
It categorized the computers with the allowed IPs as allowed, while the one which was not set as allowed of IP (41.89.64.60) was marked as intruder, as shown in the figure 5 below 


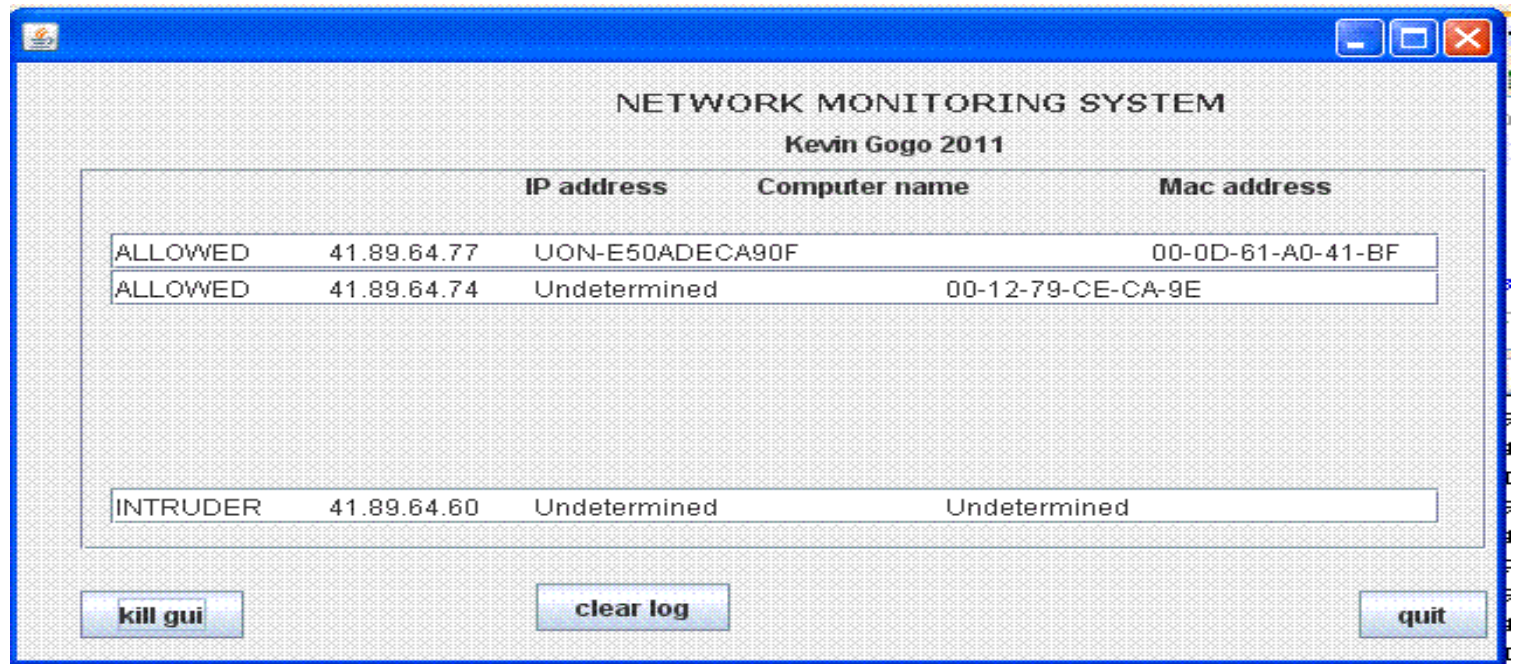

Figure 5 - Systems GUI showing results when there is network with allowed IPs entered

\subsection{Testing the System when Connected to a Network with both Static and Dynamic IPs}

The agent was run on one network with 9 computers with some computers having static IP address it identified all the 9 computers and classified them accordingly.
It also managed to classify the IPs that is in the allowed file (192.168.1.100) as allowed IPs, as shown in the figure 6 below:-

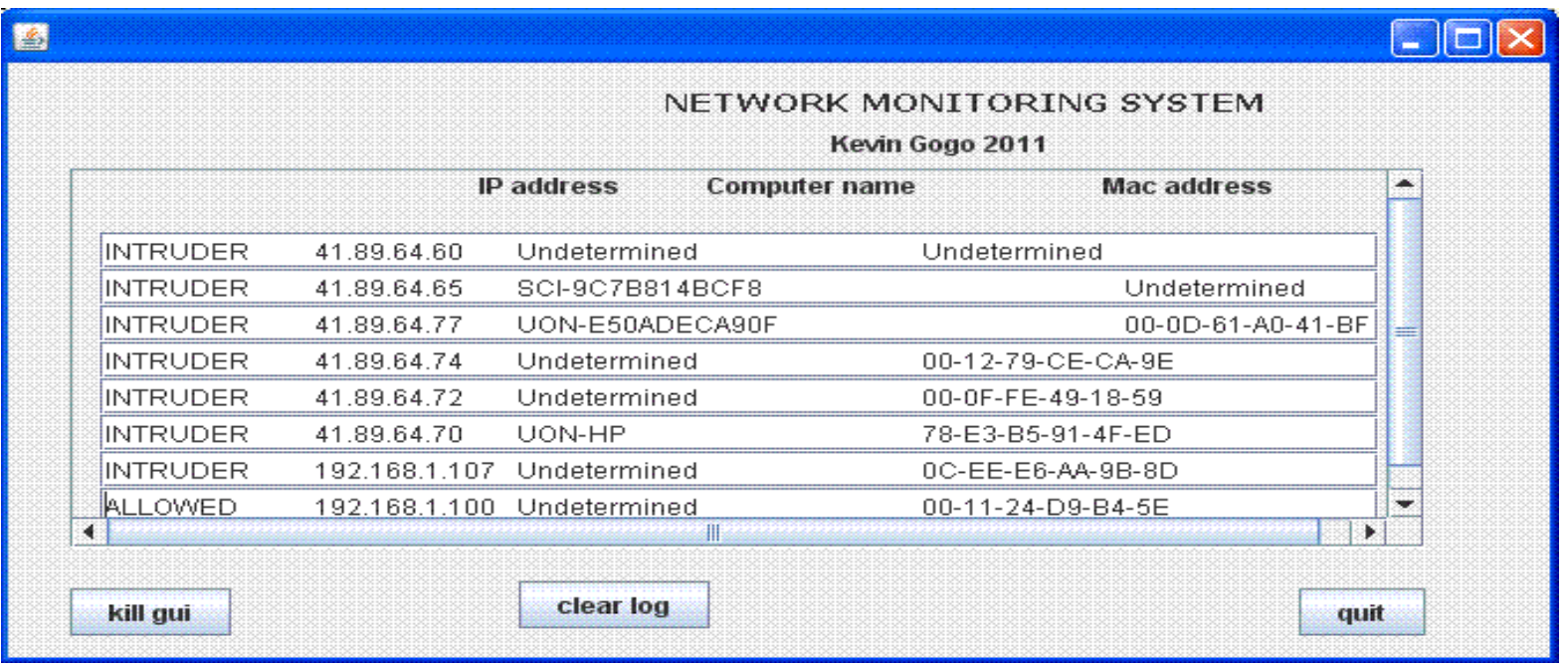

Figure 6 - System GUI showing results when networks changed as system runs

\section{CONCLUSION AND RECOMMENDATIONS}

\subsection{Conclusion}

The main objective of the mobile agents that we have developed was to relay the fundamental details of all the computers connected on the Wi-Fi P2P network. These computer network details are:- computers IP addresses, computer names and the Mac addresses. The agent was developed and tested on various Wi-Fi P2P networks as well as the cabled networks. The mobile agent successfully achieved its intended objectives, but with some slight challenges in others. It worked well on networks which consisted of a few computers, especially with IP addresses following each other sequentially. The mobile agent also classified the computers found as either allowed or intruding, which will help the network provider or user to know who is piggybacking on his/her network.

The main challenge that the mobile agent is facing is time consuming. In essence to scan a single IP address the agent takes about 2 second, which implies that if the IP range is from IP 192.168.1.1 to 192.168.1.20, then the system will take about 2 minute 30 seconds inclusive of time for learning its path which is on average 1.75 minutes. In some occasions, especially when the Wi-Fi network was weak, the agent returned a few IP addresses with an accuracy of about $80 \%$ of the total scanned IP addresses. In future we expect the system to be improved further to encompass the recommendations given below.

\subsection{Recommendations}

The system managed to solve a greater part of the problem which was at hand. We hereby recommend that in future the system could be further developed to include or improve in the following activities: -.

- Reducing the time it takes the multiagent to go through a P2P Wi-Fi network and return a substantive network detail without a miss

- Detects your internal and external IP addresses.

- Retrieves currently logged-on users, configured user accounts and uptime. 
- Supports Wake-On-LAN, remote shutdown and sending network messages.

\section{ACKNOWLEDGEMENTS}

I acknowledge the entire teaching staff of the University of Nairobi - School of Computing and Informatics, for taking their time in feeding us with the precious knowledge which enabled us to come up with this project. Yet still I could not forget my family members Babra, Shirleeen and my wife Elizabeth for offering me the emotional strength during the times of writing and carrying on with this project.

\section{REFERENCES}

[1] Chauhan D., 1997. JAFMAS: A Java-Based Agent Framework for Multiagent Systems Development and Implementation, master's thesis. Electrical Engineering and Computer Science Dept., Univ. of Cincinnati, Cincinnati.

[2] El-Rewini H., Abd-El-Barr M. 2005. Fundamentals of computer organization and architecture. A John Wiley \& sons, Inc.

[3] Joey P. 2010. Peer to peer configuration, [online]. available from http://www.wifinotes.com/computernetworks/how-to-configure-network.html [accessed on10th December 2014]

[4] Kothari C.R. 2004. Research methodology, $2^{\text {nd }}$ ed., New Delhi: New Age Int. (P) Ltd.

[5] Li J. C, 2001. Mobile agents system, a tutorial report of University of Calgary. Available on http://people.ucalgary.ca/ far/Lectures/SENG697/PD F/tutorials/2001/Mobile_Agents2.pdf [accessed on10th December 2014]

[6] Michael W. 2002. An Introduction to Multiagent Systems. John Willey \& Sons, Ltd.

[7] Michiardi, P. Molva, R. 2003 "Ad hoc networks security, ” IEEE Press Wiley, New York,

[8] Microsoft, 2006. Microsoft Data Network (MSDN) knowledge base. Viewed on $12^{\text {th }}$ December 2014 <http:// www.microsoft.com>
[9] Nwana H.S. 1996. 'Software agents: An Overview', in Knowledge Engineering Review.

[10] Odell J., Parunak H., and Bauer B. 2000. Extending UML for agents. In Proceedings of the AgentOriented Information Systems Workshop at the 17th National conference on Artificial Intelligence.

[11] Opiyo Elisha, Erick Ayienga, Katherine Getao, Bernard Manderick, Okello-Odongo, and Ann Nowé 2006. "Computing Research Challenges and Opportunities with Grid Computing”, in Ddembe W. and Baryamureeba V. (eds); Measuring Computing Research Excellence and Vitality, Fountain publishers, Kampala. Online version available at http://cit.mak.ac.ug/iccir/downloads/SREC05.pdf

[12] Phillips M. 2010. Mobile and Ambient Agents in the Internet and in the Electronic Corposants', available from

http://www.limsi.fr/ jps/enseignement/examsma/200 4/BHATTI/index.htm\#Mobile\%20Agents, [accessed on10th December 2014]

[13] Rapaport T.S., 2002. Wireless Communications Principles and Practice, Prentice Hall.

[14] Tarau, P. Dahl, V. De Bosschere, K. 1997. High level logic programming tools for remote execution, mobile code and agents, [accessed on 8th December 2014] available

at http://clement.info.umoncton.ca/html/remote/art.ht $\mathrm{ml}$

[15] Tesfatsion, L. 2001b. Agent-based modeling of evolutionary economic systems, IEEE Transactions on Evolutionary Computation, vol. 5, pp. 1-6.

[16] Wooldridge M. 2004. An Introduction to Multi-Agent Systems, Wiley.

[17] Yang, H. Luo, H. Ye, F. Lu, S. Zhang, L. 2004. "Security in mobile ad hoc networks: challenges and solutions," In proc. IEE Wireless Communication, UCLA, Los Angeles, CA, USA; volume- 11, ISSN: 1536-1284

[18] http://en.wikipedia.org/wiki/Simple_Network_Manage ment_Protocol [accessed on 8th December 2014] 